\title{
Les revenus des cures du diocèse de Nicolet, 1885-1904
}

\section{Jean Roy}

Volume 52, 1985

URI : https://id.erudit.org/iderudit/1007002ar

DOI : https://doi.org/10.7202/1007002ar

Aller au sommaire du numéro

Éditeur(s)

Les Éditions Historia Ecclesiæ Catholicæ Canadensis Inc.

ISSN

0318-6172 (imprimé)

1927-7067 (numérique)

Découvrir la revue

Citer cet article

Roy, J. (1985). Les revenus des cures du diocèse de Nicolet, 1885-1904. Sessions d'étude - Société canadienne d'histoire de l'Église catholique, 52, 51-67.

https://doi.org/10.7202/1007002ar

\section{Résumé de l'article}

Les changements apportés à l'agriculture, le développement de l'industrie rurale, les migrations régionales ainsi que la consolidation des villages causèrent l'affaiblissement des revenus curiaux. À la demande des curés, l'évêque Elphège Gravel intervint pour redresser la situation en leur faveur. Il conclut la délicate opération le 11 septembre 1891, en fixant les grandes lignes d'un règlement sur la dîme, le supplément et la capitation, qui faisait reposer sur tous les fidèles, tant les cultivateurs que les ouvriers, la responsabilité d'entretenir les curés. Toutefois, il est permis de se demander si la hausse du tarif du casuel, uniformément appliquée, n’a pas été un facteur décisif. Sans elle, le but aurait-il été atteint?
Tous droits réservés @ Les Éditions Historia Ecclesiæ Catholicæ Canadensis Inc., 1985
Ce document est protégé par la loi sur le droit d'auteur. L'utilisation des services d'Érudit (y compris la reproduction) est assujettie à sa politique d'utilisation que vous pouvez consulter en ligne.

https://apropos.erudit.org/fr/usagers/politique-dutilisation/ 


\title{
Les revenus des cures du diocèse de Nicolet, 1885-1904
}

\author{
Jean Roy \\ Département des sciences humaines \\ Université du Québec à Trois-Rivières \\ RÉSUMÉ
}

Les changements apportés à l'agriculture, le développement de l'industrie rurale, les migrations régionales ainsi que la consolidation des villages causèrent l'affaiblissement des revenus curiaux. À la demande des curés, l'évêque Elphège Gravel intervint pour redresser la situation en leur faveur. Il conclut la délicate opération le 11 septembre 1891, en fixant les grandes lignes d'un règlement sur la dîme, le supplément et la capitation, qui faisait reposer sur tous les fidèles, tant les cultivateurs que les ouvriers, la responsabilité d'entretenir les curés. Toutefois, il est permis de se demander si la hausse du tarif du casuel, uniformément appliquée, n'a pas été un facteur décisif. Sans elle, le but aurait-il été atteint?

Au cours du dernier quart du XIX ${ }^{\mathrm{e}}$ siècle, l'épiscopat québécois eut à se préoccuper de la situation financière du clergé paroissial. Celui-ci se plaignait, en effet, de la chute de ses revenus, soulevant en particulier le problème posé par l'insuffisance de la dîme. Historiquement la première de ses ressources financières, elle était demeurée à la base du régime de la «rétribution des curés» ruraux, bien que des transformations profondes soient intervenues et se produisaient encore au sein des campagnes. Les changements apportés à l'agriculture, l'industrialisation du monde rural, la 
mobilité et la formation des villages avaient des incidences directes sur la condition matérielle des curés. Aussi, requéraient-ils l'intervention des évêques pour modifier les principes qui régissaient la rémunération des curés. Il en était ainsi dans le nouveau diocèse de Nicolet, créé en 1885. Or, pour l'évêque, la question prenait une importance accrue par les rapports qu'il entretenait avec son clergé. Issu d'un diocèse étranger, vraisemblablement choisi par $\mathbf{M}^{\mathrm{gr}}$ Taschereau, Elphège Gravel paraît avoir rencontré beaucoup de difficultés dans ses relations avec les prêtres du diocèse. La prudence exigeait donc de ne rien précipiter, de poursuivre la politique déjà engagée. $\mathrm{M}^{\mathrm{gr}}$ Gravel travailla dans ce sens, tâchant de ne rien imposer avant que la décision ne parut acceptée de tous. Comme ses collègues, l'évêque émit en 1891, une lettre pastorale dont le but était d'améliorer les revenus curiaux. A-t-il atteint son objectif? Le moyen utilisé fut-il efficace? Les réponses à ces questions exigent, en premier lieu, que l'on esquisse un portrait du diocèse. Utilisant plus particulièrement des informations fournies par les curés, il est possible d'évaluer l'ampleur du problème, d'examiner les revenus des cures et de mesurer l'impact des mesures administratives. Avant de traiter de ces divers points, précisons enfin que l'enquête couvre les quelque vingt années de l'épiscopat de $\mathrm{M}^{\mathrm{gr}}$ Gravel, les débordant parfois en amont ${ }^{\prime}$.

\section{Les populations diocésaine et paroissiale}

Depuis un peu plus de trente ans, on taillait à même les grands diocèses de Québec et de Montréal pour ériger de nouvelles circonscriptions religieuses. D'étendues variables, pensons à Trois-Rivières en 1852, à Rimouski en 1867 et à Chicoutimi en 1878, elles différaient également par le nombre des paroisses. En 1887, les diocèses trifluvien, rimouskois et chicoutimien regroupaient respectivement 31,68 et 32 paroisses. Nicolet qui, en 1885, en comptait 49, se classait avec Sherbrooke, parmi les diocèses de taille moyenne. Cet indicateur ne suffit cependant pas, à lui seul, à le caractériser. Nuançons avec des statistiques de sa population. Les 79512 fidèles qui en faisaient partie en 1885, faisaient de Nicolet le troisième plus important diocèse rural de la province, après Saint-Hyacinthe et Rimouski, mais avant Sherbrooke et Trois-Rivières. Il était cependant le seul dont toute l'étendue était habitée. Déjà en 1875, des paroisses constituées traçaient son coutour. Des diocésains résidaient sur son pourtour. Le diocèse n'allait pas se développer que de l'intérieur. C'est ainsi que, hor-

\footnotetext{
${ }^{1}$ Les sources de l'étude furent tirées des Archives de l'évêché de Nicolet: les rapports annuels des curés, les procès-verbaux des visites pastorales, les registres des insinuations ecclésiastiques et les registres des lettres pour la période 1885-1904 ainsi que les rapports annuels des curés, 1875-1885.
} 
mis la paroisse de Sainte-Christine, toutes les nouvelles paroisses nâ̂traient des démenbrements des paroisses anciennes du diocèse ${ }^{2}$. En 1904, le diocèse de Nicolet épousait les formes et englobait les limites des comtés de Nicolet et d'Arthabaska dans sa partie est, celles des comtés de Yamaska et de Drummond dans sa partie ouest, avec en plus, une bande de territoire empruntée aux comtés de Bagot et de Shefford, soudée à la limite sud du comté de Drummond. (Voir carte).

L'occupation de la totalité du territoire diocésain ne reflète cependant pas la colonisation active, les défrichements qui entraînèrent la création de 12 paroisses entre 1885 et 1905 , alors qu'on estimait, à l'origine, que le nombre des paroisses ne pouvait augmenter de plus de 3 ou $4^{3}$. Au début de son épiscopat, en 1904, Hermann Brunault, second évêque de Nicolet, avait en charge 62 paroisses et une mission sur le territoire desquelles vivait une population répartie en 88000 catholiques et environ 1558 protestants $(-2 \%)$. Ceux-ci avaient vu fondre leurs effectifs depuis 1885 , allors qu'ils étaient près de 2700 . Majoritairement localisés à la frontière sud du diocèse, dans les cantons de Kingsey et de Durham, ils habitaient également les cantons de Warwick et de Tingwick, de même qu'il s'en trouvait un bon nombre dans la paroisse de Saint-Frédéric. Vingt ans plus tard, leur concentration dans les cantons frontaliers du sud-ouest s'était accentuée, prenant une grande importance à Saint-Fulgence. Là, la population protestante, 900 personnes, composait $55 \%$ de la population totale de la paroisse. C'était aussi plus de la moitié de l'effectif total protestant du diocèse. À l'opposé, les catholiques avaient accru leur nombre de 10000 dans le même laps de temps. Doit-on faire remarquer que c'est peu, compte tenu des taux de natalité élevés de l'époque. Le responsable était, bien sûr, l'émigration, source de nombreux soucis.

Les autorités diocésaines cherchaient à se renseigner sur le déplacement de la population, car il ne manquait pas d'affecter l'administration des paroisses. Ainsi, à Saint-Cyrille où Elphège Gravel effectuait sa visite pastorale au mois de juillet 1895, il admit facilement que l'église était devenu trop exiguë. Mais deux raisons l'incitaient à ne pas permettre l'agrandissement souhaité: le démembrement prochain de la paroisse au profit de la création de la paroisse Notre-Dame-du-Bon-Conseil qui lui enlèverait quelques familles; et surtout, la fermeture anticipée d'une industrie du bois qui provoquerait, selon lui, le départ d'une cinquantaine de familles à la recherche de travail. Ailleurs, ce fut l'agriculture qui déclen-

\footnotetext{
${ }^{2}$ Une partie du territoire avait appartenu à la juridiction religieuse de Saint-Hyacinthe et relevait, au civil, du comté de Bagot.

${ }^{3}$ Archives du Séminaire de Nicolet (désormais ASN), Division du diocèse $\mathrm{ZZ} \mathrm{ms}$ «Notes demandées par $\mathrm{M}^{\mathrm{gr}}$ l'Archevêque de Québec», mai 1883, 6 pages.
} 


\section{Le diocèse de Nicolet}

\section{à la fin de l'épiscopat d'Elphège Gravel,}

\section{4}

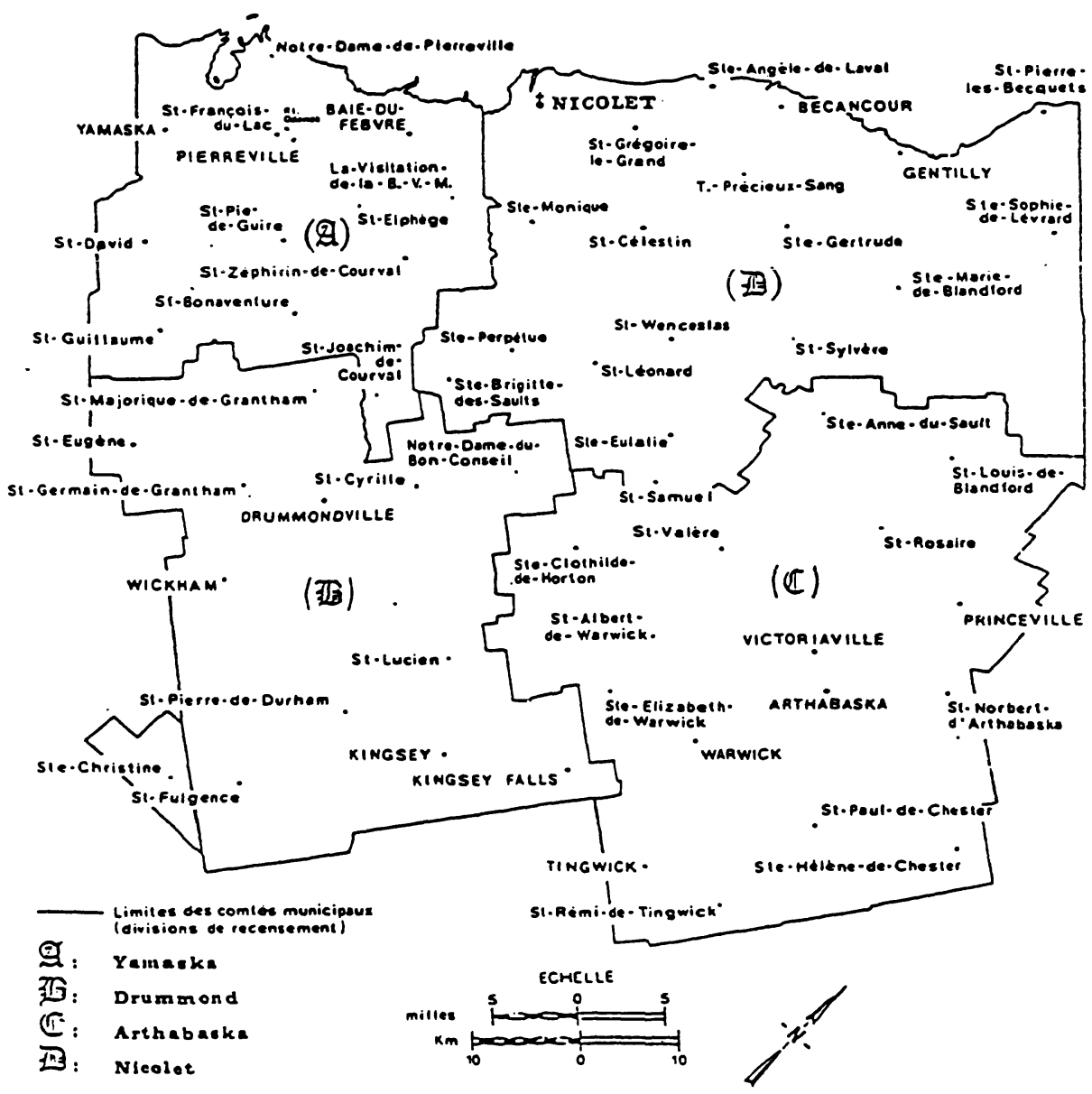


cha le mouvement migratoire. Dans le procès-verbal de la visite qu il fit dans la paroisse de Saint-Pierre-les-Becquets, l'évêque nota. au mois de juillet 1893, que 73 familles avaient quitté leur foyer au cours des trois dernières années. Il comptait sur l'amélioration de l'agriculture pour enrayer le mouvement. Or, la mobilité continua d'être très grande. Elle touchait un grand nombre de familles qui partaient temporairement ou définitivement, ainsi qu'une grande quantité de célibataires, garçons et filles (tableau 1).

\section{Tableau 1}

La mobilité par comté dans le diocèse de Nicolet, 1885-1905

\begin{tabular}{|c|c|c|c|c|c|c|c|c|c|c|}
\hline & \multicolumn{2}{|c|}{ Yamaska } & \multicolumn{2}{|c|}{ Drummond } & \multicolumn{2}{|c|}{ Nicolet } & \multicolumn{2}{|c|}{ Arthabaska } & \multicolumn{2}{|c|}{ Totaux } \\
\hline & 1885 & 1904 & 1885 & 1904 & 1885 & 1904 & 1885 & 1904 & 1885 & 1904 \\
\hline $\begin{array}{l}\text { Familles qui } \\
\text { ont quitté la } \\
\text { paroisse }\end{array}$ & 54 & 122 & 78 & 76 & 59 & 109 & 52 & 97 & 243 & 404 \\
\hline $\begin{array}{l}\text { Familles de } \\
\text { retour }\end{array}$ & 69 & 62 & 140 & 72 & 64 & 51 & 100 & 123 & 373 & 308 \\
\hline Solde & 15 & -60 & 62 & -4 & 5 & -58 & 48 & 26 & 130 & -96 \\
\hline $\begin{array}{l}\text { Jeunes gens } \\
\text { qui ont quitté } \\
\text { la paroisse }\end{array}$ & 94 & 142 & 102 & 13 & 259 & 82 & 113 & 149 & 568 & 386 \\
\hline
\end{tabular}

Source: Les rapports annuels des curés des années 1885 et 1904.

En 1885,616 familles et 568 «jeunes gens» marquèrent le mouvement qui, en 1904, était encore plus profond avec 712 familles et 386 célibataires. La mobilité des familles fut particulièrement grande dans les comtés de Drummond, en 1885, et d'Arthabaska, en 1904, alors que le comté de Nicolet fournit, en 1885, une impressionnante cohorte de célibataires. Certes, tous les migrants ne sortaient pas du diocèse. Le curé de Saint-Grégoire écrivit dans son rapport de 1885 qu'à «l'exception d'une (famille) qui est allée dans le diocèse de Sherbrooke, elles (les 6 autres) sont allées dans les paroisses voisines». Cette année-là, une trentaine de familles et 40 à 50 jeunes gens quittèrent la paroisse de Saint-Guillaume. Tous se dirigèrent vers les États-Unis. De retour d'un voyage à Manchester, le curé de Sainte-Monique nota dans son rapport de l'année 1885 qu'il $\mathrm{y}$ avait environ trois cents individus provenant de sa paroisse. Il ajouta que «les uns reviennent et d'autres retournent». Les statistiques des seules années 1885 et 1904 suggèrent donc l'existence d'un phénomène marquant. Si la mobilité ne fut pas entièrement dirigée vers l'extérieur, elle 
explique, en bonne partie, la faible progression démographique au cours des deux décennies. La mesure au niveau des comtés permet de préciser davantage l'ampleur du fait migratoire en éliminant les fluctuations des populations paroissiales imputables à des démembrements (tableau 2).

\section{Tableau 2}

\section{La population du diocèse, par comtè}

$\begin{array}{lrrr} & \mathbf{1 8 8 5} & \mathbf{1 9 0 4} & \text { Écart } \\ \text { Yamaska } & 20177 & 20947 & -770 \\ \text { Drummond } & 14705 & 17331 & 2626 \\ \text { Nicolet } & 25164 & 27671 & 2507 \\ \text { Arthabaska } & 19466 & 23639 & 4173 \\ \text { Total } & 79512 & 89588 & 10076\end{array}$

Source: Les rapports annuels des curés 1885, 1904.

Alors que le comté d'Arthabaska augmenta sa population de 4173 habitants, celui d'Yamaska accusa une perte de 770 personnes. Au cours des deux décennies, les deux autres juridictions, Nicolet et Drummond, enregistrèrent une croissance assez semblable bien que la taille des populations de 1885 était très inégale. En 1885, l'écart qui séparait les comtés du nord et du sud était de 11170 personnes, en faveur des comtés du nord; en 1904, la différence était réduite à 7 648. Bien évidemment cela traduit les fluctuations importantes au niveau des paroisses. Comme dans l'estimation des revenus, seule importe la taille brute des populations paroissiales, il n'y a pas lieu d'insister sur les causes. Dans le comté d'Yamaska, les paroisses de Saint-Thomas-de-Pierreville et de Saint-Antoine-de-la-Baiedu-Febvre connurent des baisses de population très considérables: respectivement 1302 et 638 habitants. Seule la paroisse de Saint-Bonaventure accrut notablement sa population de 414 personnes. Aucune paroisse du comté de Nicolet n'accusa de telles pertes, quoiqu'elles furent de l'ordre de 500 habitants à Bécancour et à Saint-Grégoire et de 300 habitants à Gentilly. À l'opposé, des paroisses progressèrent de plus de 500 personnes: Sainte-Perpétue, Saint-Pierre-les-Becquets; d'autres moins: 400 pérsonnes à Nicolet, par exemple. Dans le comté de Drummond, les paroisses de Saint-Fulgence, Saint-Frédéric et Saint-Germain décrûrent de 991, 654 et 506 habitants respectivement, alors que les paroisses de Saint-Cyrille et de Saint-Eugène crûrent de 991 et 433 habitants. Tournons-nous enfin vers le comté d'Arthabaska où la paroisse de Sainte-Victoire grossit de 1383 fidèles et celle de Sainte-Clothilde de 607. Là, les pertes furent rares: il n'y eut que la paroisse de Sainte-Hélène-de-Chester avec 340 habitants. 


\section{Tableau 3}

\section{Les populations paroissiales en 1885 et 1904}

$\begin{array}{llrr} & \text { Nombre d'habitants } & \text { Nombre de paroisses } \\ & \text { catholiques et protestants } & \mathbf{1 8 8 5} & \mathbf{1 9 0 4} \\ \text { I } & 3000 \text { et plus } & 3 & 2 \\ \text { II } & 2000-2999 & 14 & 12 \\ \text { III } & 1400-1999 & 11 & 16 \\ \text { IV } & 800-1399 & 13 & 19 \\ \text { V } & 799 \text { et moins } & \frac{8}{49} & \underline{13} \\ & & & 62\end{array}$

Source: Les rapports annuels des curés.

Ce bref survol confirme, en la précisant, l'analyse du géographe Raoul Blanchard ${ }^{4}$. Il avait observé l'accroissement rapide des populations des paroisses du lac Saint-Pierre au XIX ${ }^{\mathrm{e}}$ siècle, le ralentissement à partir de 1875 , l'arrêt vers 1891 . Il estimait également les pertes à pas moins de 6000 habitants en 1901, soit 9\%. Il affirmait, de plus, l'existence d'un dépeuplement rural alors que «villes et bourgades» avaient continué à augmenter. À première vue, l'idée du dépeuplement semble entrer en contradiction avec l'étalement des populations paroissiales sur le territoire: que ce soit par la présence de nombreuses paroisses moyennes (catégorie III et IV du tableau 3) ou encore par l'augmentation des petites paroisses. En 1904, avec ses nombreuses paroisses de moins de 2000 habitants, le comté de Nicolet était principalement composé de paroisses de taille moyenne. Le comté regroupait moins de très grosses paroisses qu'en 1885. Un semblable glissement s'était produit dans le comté d'Yamaska où les paroisses de Saint-Thomas-de-Pierreville et de Saint-Guillaume, toutes deux de plus de 3000 habitants, changèrent de catégorie. Elles consolidèrent leur nouveau groupe d'appartenance. Ensemble, elles donnaient une forte densité à cette partie du diocèse. Dans Drummond, si on excepte le cas de la paroisse de Saint-Cyrille qui progressa de façon remarquable, le classement ne laisse pas voir de changements très importants, comme ce le fut dans le comté d'Arthabaska. La paroisse de Sainte-Victoire parvint à prendre place dans le groupe des très grosses paroisses, bien appuyée par les localités voisines de Saint-Eusèbe, de Saint-Christophe, et de SaintMédard. À l'opposé, 10 paroisses, le plus fort total, avaient moins de 1400 habitants.

\footnotetext{
${ }^{4}$ Raoul Blanchard, Le Centre du Canada français, province de Québec, Montréal, Beauchemin, 1947.
} 
Tableau 4

\section{Les emplacitaires}

1885

1904

\begin{tabular}{|c|c|c|c|c|c|c|c|c|c|c|}
\hline & $\begin{array}{l}\text { Nombre } \\
\text { total des } \\
\text { familles }\end{array}$ & $\begin{array}{r}\text { Familles } \\
\text { emplacitaires }\end{array}$ & $\%$ & $\begin{array}{l}\text { Familles } \\
\text { au village }\end{array}$ & $\%$ & $\begin{array}{l}\text { Nombre } \\
\text { total des } \\
\text { familles }\end{array}$ & $\begin{array}{r}\text { Familles } \\
\text { emplacitaires }\end{array}$ & $\%$ & $\begin{array}{l}\text { Familles } \\
\text { au village }\end{array}$ & $\%$ \\
\hline Nicolet & 3908 & 911 & 23,3 & $643^{1}$ & 16,4 & 4175 & 865 & 20,7 & 696 & 16,6 \\
\hline Yamaska & 3393 & $955^{2}$ & 28,1 & 679 & 20 & 3437 & 1414 & 40,7 & 925 & 26,9 \\
\hline Arthabaska & 2994 & 819 & 27,3 & 683 & 22,8 & 4112 & 1212 & 29,4 & 1215 & 29,5 \\
\hline TOTAUX & 12547 & 3470 & 27,6 & 2723 & 21,7 & 14685 & 4299 & $2.9,2$ & 3646 & 24,8 \\
\hline
\end{tabular}

'La notion de village n'est pas définie. La question est la suivante: $\mathrm{Y}$ a-t-il un village auprès de l'église? Quel est le nombre de familles'?

${ }^{2}$ Manquent, les statistiques sur la paroisse de Saint-David.

Source: Les rapports annuels des curés. 
Cette lecture de la distribution des populations paroissiales a permis de relever quelques contrastes et d'identifier des zones de peuplement plus intense, en même temps qu'elle a donné l'occasion d'observer une certaine évolution que l'on peut chercher à mieux cerner. L'affinement des observations va emprunter la voie d'une distinction entre cultivateur et emplacitaire. Certes, le concept d'emplacitaire pose certains problèmes à son utilisateur et mon intention n'est pas d'en discuter. Pour les besoins de cette étude, il a paru suffisant d'identifier clairement l'un des deux statuts: le cultivateur. Quant à l'emplacitaire, il habitait un emplacement, quelque part, dans la paroisse. Ce pouvait être dans le village, à proximité de l'église, mais ce pouvait être également ailleurs, dans un rang, dans un hameau, situé sur le territoire de la paroisse.

L'analyse des statistiques fournies par les curés est sur ce plan très révélatrice (tableau 4). À l'échelle des comtés, rien n'est plus remarquable que la progression des emplacitaires dans Yamaska. Certes, en 1885, le comté avait déjà, en chiffres absolus, le plus grand nombre de familles emplacitaires (955) bien qu'en pourcentage, il cédait la première place au comté de Drummond, 34,8 contre 28,1. En 1904, les 1414 familles emplacitaires de Yamaska représentaient $40 \%$ de toutes les familles. Elles étaient nettement majoritaires à Saint-Thomas, à Notre-Dame-dePierreville et à Saint-François-du-Lac. Elles constituaient presqu' une moitié à la Baie-du-Febvre, alors que dans la paroisse de Saint-Michel-deYamaska, elles se rapprochaient des $30 \%$. Le second comté en importance était Arthabaska, avec, en tête, la paroisse de Sainte-Victoire dont les deux tiers des familles étaient emplacitaires. Venaient ensuite les paroisses de Saint-Christophe et Saint-Eusèbe. La paroisse de Saint-Médard présentait un contingent de 120 familles. Rien de tel dans le comté de Drummond, sauf à Saint-Frédéric, qui avait accusé une baisse notable, et à SaintGermain-de-Grantham. Le comté de Nicolet se situait en deçà de la moyenne diocésaine. Là, hormis les paroisses de Saint-Jean-Baptiste-deNicolet, Saint-Édouard-de-Gentilly et Sainte-Angèle-de-Laval - dans celle-ci les familles emplacitaires, au nombre de 102 , composaient $60 \%$ de toutes les familles - aucune autre paroisse ne regroupait plus de $25 \%$ de familles emplacitaires.

En 1885 , près de $28 \%$ de toutes les familles étaient emplacitaires. Vingt ans plus tard, cette proportion avait augmenté principalement dans les paroisses situées au nord du comté d'Yamaska - dans les paroisses du lac Saint-Pierre, pour reprendre le découpage spatial utilisé par Raoul Blanchard, et autour de Victoriaville. 


\section{La structure des revenus curiaux}

La lettre pastorale du 11 septembre $1891^{5}$, sur la dîme, prenait donc place dans un contexte défini par une faible augmentation de la population qui, par ailleurs, avait diminué dans certaines paroisses, alors que le nombre d'emplacitaires avait progressé. L'évêque souhaitait mettre un point final aux interventions qui s'étaient succédées depuis le 19 mars $1889^{6}$ sur cette question. Il notait déjà. dans la lettre circulaire signée à cette date, que ia cuiture du foin ei l'exiension des pâturages avâient boùleverersé l'agriculture et affecté les revenus curiaux encore largement dépendants du produit de la dîme du grain. Il faisait également remarquer que l'agriculture n'occupait plus qu'une partie de la population, sans autrement préciser que c'était là un effet des progrès de l'industrie rurale. Le but de l'évêque était simple: rendre tous les fidèles responsables de la condition matérielle du clergé paroissial afin de compléter les revenus de la dîme, mais par quel moyen' L'évêque demanda aux curés de lui faire connaître leur opinion sur le sujet. Tout indique qu'ils s'empressèrent de donner leur avis. Chacun rélléchit sur sa rémunération et suggéra la solution jugćc convenable. La diversité des opinions rendit impossible l'élaboration d'une mesure générale et convainquit l'évêque que le diocèse n'était pas prêt à accepter un «supplément uniforme». Toutefois, il souhaitait parvenir à une solution avant l'été 1890 . Finalement, il invitait les curés à discuter de cette question entre eux. Le $1^{\text {er }}$ mai $1890, M^{\text {gr }}$ Gravel présenta les grandes lignes du règlement qu'il se proposait d'adopter au cours de l'été. Près de huit mois plus tard, le 11 septembre 1891, l'évêque déposa enfin son règlement. Pour l'essentiel, il reprenait les arguments contenus dans les circulaires précédentes. L'extension de la culture du foin sur les meilleures terres et la place prise par les pâturages au détriment de la culture des grains faisaient en sorte que les plus riches payaient moins que d'autres. Il ajoutait enfin que les familles des villages, privilégiées quant aux services qui leur étaient offerts, échappaient à la dîme. Aussi, jugeait-il qu'il était temps d'intervenir. Les nombreux témoignages qu'il avait recueillis l'incitaient à croire que le règlement serait bien reçu. La lettre pastorale faisait également une courte histoire des interventions épiscopales. L'évêque de Trois-Rivières avait cherché, avant 1885, à équilibrer les obligations des fidèles, et en conséquence, il avait pris plusieurs mesures, toutes locales cependant. Quant à lui, il avait poursuivi dans ce sens, tout en consultant les intéressés, préparant ainsi les esprits à un règlement général qui parais-

\footnotetext{
5 «Lettre pastorale de Monseigneur l'évêque de Nicolet sur la dîme», Mandements des évêques de Nicolet (désormais MEN), vol. 1, $\mathrm{n}^{\circ} 40$.

${ }^{6}$ Circulaire au clergé. $n^{\circ} 26,19$ mars 1889, «Rétribution des curés»; Circulaire au clergé, $\mathrm{n}^{\circ} 30,24$ novembre 1889, "Supplément»; Circulaire au clergé, n 35, 1 mai 1896, «Supplément à la dîme», dans les $M E N$, volume 1 .
} 
sait s'imposer. Les délais apportés à sa rédaction témoignent des difficultés rencontrées. En apparence, elles n'étaient pas toutes levées. C'est du moins ce qui ressort de la lettre circulaire qui ascompagnait la lettre pastorale et dans laquelle l'évêque reconnaissait que le règlement était susceptible de contrarier les curés auxquels il avait imposé des correctifs particuliers. Afin d'éviter tout conflit, les curés pourraient ne pas publier le règlement, sur autorisation de l'évêché. Elphège Gravel n'avait pas' la tâche facile: il existait une variété de situations.

\section{a) La taille des revenus curiaux}

Le texte du règlement laisse supposer la présence d'un large éventail des conditions économiques paroissiales et, comme corrolaire, l'existence d'une non moins grande fourchette de revenus curiaux. Afin d'en arriver à une lecture de l'évolution des revenus curiaux et, pour parvenir à déceler l'impact des mesures, le point a été fait à trois époques: en 1885 et en 1904 , et vers $1875-1883^{7}$.

Le projet de création d'un nouveau diocèse sur la rive droite du SaintLaurent imposait aux promoteurs de démontrer qu'ils ne mettaient pas en danger la viabilité du diocèse amputé. L'opération donna lieu à une collecte d'informations d'où émanent des «Notes sur les deux parties du diocèse des Trois-Rivières, le Nord et le Sud», préparées à l'intention de $\mathrm{M}^{\mathrm{gr}}$ Taschereau, au mois de mars 1883 . Le ou les auteurs du document répartissaient les paroisses de la rive sud en trois classes, d'après leurs revenus. La première regroupait 16 paroisses qui, à l'exception des paroisses de Saint-Eusèbe, Saint-Christophe et Saint-Norbert, appartenaient aux comtés de Nicolet (6 paroisses) et Yamaska (7 paroisses). À l'opposé, le comté d'Arthabaska comptait le plus de cures de troisième classe, 6; alors que celui d'Yamaska n'en avait qu'une seule, la paroisse de Saint-Pie-deGuire.

\footnotetext{
${ }^{7} A S N$. Division du diocèse $\mathrm{ZZ}$ ms «Notes demandées par M"r l'Archevêque dc Québec», mai 1883. Les rédacteurs n'ont livré aucune statistique des revenus. Mais, des recoupements laissent penser que le document fut fabriqué à l'aide de la hiérarchie des revenus entre 1875 et 1883 . C'est ainsi que le point fut fait pour cette période que nous situons autour de 1875 .
} 


\section{Tableau 5}

\section{Classement des cures en 1883}

$\begin{array}{lrrr}\text { Comté } & \mathbf{1}^{\mathbf{r e}} \text { classe } & \mathbf{2}^{\mathbf{e}} \text { classe } & \mathbf{3}^{\mathbf{e}} \text { classe } \\ \text { Yamaska } & 7 & 1 & 1 \\ \text { Drummond } & 0 & 5 & 3 \\ \text { Nicolet } & 6 & 5 & 4 \\ \text { Árthabaskia } & 3 & 4 & 6\end{array}$

Source: ZZ MS A.S.N. Divisions dụ diocèse. «Notes demandées...», mai 1883.

\section{Tableau 6}

\section{La taille des revenus curiaux}

\begin{tabular}{|c|c|c|c|}
\hline en dollars & $1875-1883$ & 1885 & 1904 \\
\hline plus de 2000 & & 1 & 4 \\
\hline $1500-1999$ & 1 & 2 & 11 \\
\hline $1000-1499$ & 7 & 13 & 10 \\
\hline $600-999$ & 9 & 12 & 21 \\
\hline moins de 600 & 19 & 16 & 12 \\
\hline nombre de paroisses ${ }^{1}$ & 36 & 44 & 58 \\
\hline
\end{tabular}

Source: Les rapports annuels des curés.

Les commentaires seraient nombreux. Mais il faut insister principalement sur le grand nombre des cures de plus de 1000 dollars en 1904, soit 25 dont 15 fournissaient plus de 1500 dollars. À l'opposé, 12 curés recevaient moins de 600 dollars dont 9 se trouvaient dans les comtés de Drummond et d'Arthabaska. Soulignons, enfin, que 6 paroisses de cette catégorie étaient des créations postérieures à 1885 . Par rapport aux revenus de l'année 1885, la progression des revenus est évidente. Notons la réduction du nombre des cures de moins de 600 dollars, 16 à 12, et l'augmentation du nombre de paroisses dans la catégorie des $600-999$ dollars, 12 à 21 . La barre des 600 dollars est un seuil que 19 cures n'avaient pas franchi en 1875,10 d'entre elles étant même évaluées à moins de 400 doltars. La progression des revenus fut donc réelle, quelle que fut la hausse des prix qui, à elle seule, ne peut expliquer des augmentations parfois considérables. Dans certains cas, en effet, l'écart fut étonnant: ainsi dans la paroisse de Sainte-Victoire, le revenu passa de 451 à 2725 dollars; à Saint- 
Christophe, la progression fut de 300\% (700 à 2245 dollars); à SaintGermain-de-Grantham, de 900 à 1900 dollars entre 1885 et 1904. Ce sont là les hausses les plus spectaculaires. Mais d'autres augmentations n'en sont pas moins substantielles: 600 dollars à Sainte-Perpétue, 500 dollars à Sainte-Clothilde. Le comté d'Arthabaska qui n'avait que deux paroisses dans la catégorie des 1000 dollars et plus en 1885, en avait 6 deux décennies plus tard. Pour mieux saisir le cheminement parcouru, il faut ventiler les revenus curiaux.

\section{b) L'origine des revenus curiaux ${ }^{8}$}

À première vue, les revenus proviennent de quatre sources: la dîme qui correspond à la $26^{\mathrm{e}}$ partie des grains, le supplément, la capitation, le casuel. La réalité était plus complexe. Outre le fait que le curé ne distinguait pas toujours précisément le supplément de la capitation, le chercheur doit prendre en compte la présence de très nombreux cas où la fabrique intervenait, à moins que ce ne fut l'évêque lui-même, pour verser une somme qui variait selon les cas. La diversité naquit des interventions locales, probablement à la suite des demandes des curés. À cet égard, Gravel imita la politique de son prédécesseur. Parfois il reconduisit le même règlement. Au cours des années 1886, 1888, 1889, l'évêque adressa plusieurs lettres aux fidèles dans lesquelles il les invitait à soutenir leur curé, leur indiquant les moyens d'y parvenir. Le 16 novembre 1886, il écrivit aux fidèles de la paroisse de Sainte-Clothilde-de-Horton. Il leur rappela qu'en 1883, Thomas Caron, administrateur du diocèse, avait imposé un supplément de foin, de patates, et une somme en argent. Depuis cette époque, ajoutait-il, la paroisse a grandi et il importait donc d'apporter des changements propres à refléter la nouvelle situation. En conséquence, il ordonnait aux producteurs des grains de poursuivre le paiement de la dîme sans rien changer; il maintenait le supplément du foin et des patatés pour les quatre années suivantes. D'autre part, ceux dont la valeur de la dîme n'atteignait pas 2 dollars, complèteraient cette somme en argent. Quant à ceux qui ne payaient aucune dîme, ils devraient verser deux dollars. L'évêque estimait que les ajustements permettraient d'atteindre un revenu convenable. Le 27 août 1888, l'évêque imposa un supplément aux fidèles des paroisses de Saint-Valère, Saint-Wenceslas, Sainte-Perpétue, Saint-Majorique et SaintNorbert. La paroisse de Saint-Bonaventure offre l'exemple d'une paroisse où la culture du foin était pratiquée sur les meilleures terres, frustrant ainsi

\footnotetext{
8 Jean Roy et Daniel Robert ont traité de ce sujet dans un article qui porte sur les paroisses du comté de Champlain: «Les rapports annuels des curés et l'histoire des paroisses dans la seconde moitié du XIX ${ }^{e}$ siècle», Archives, vol. 16, nº 1, (juin 1984), pp. 31-53.
} 


\section{Population paroissiale et revenus curiaux, 1904}

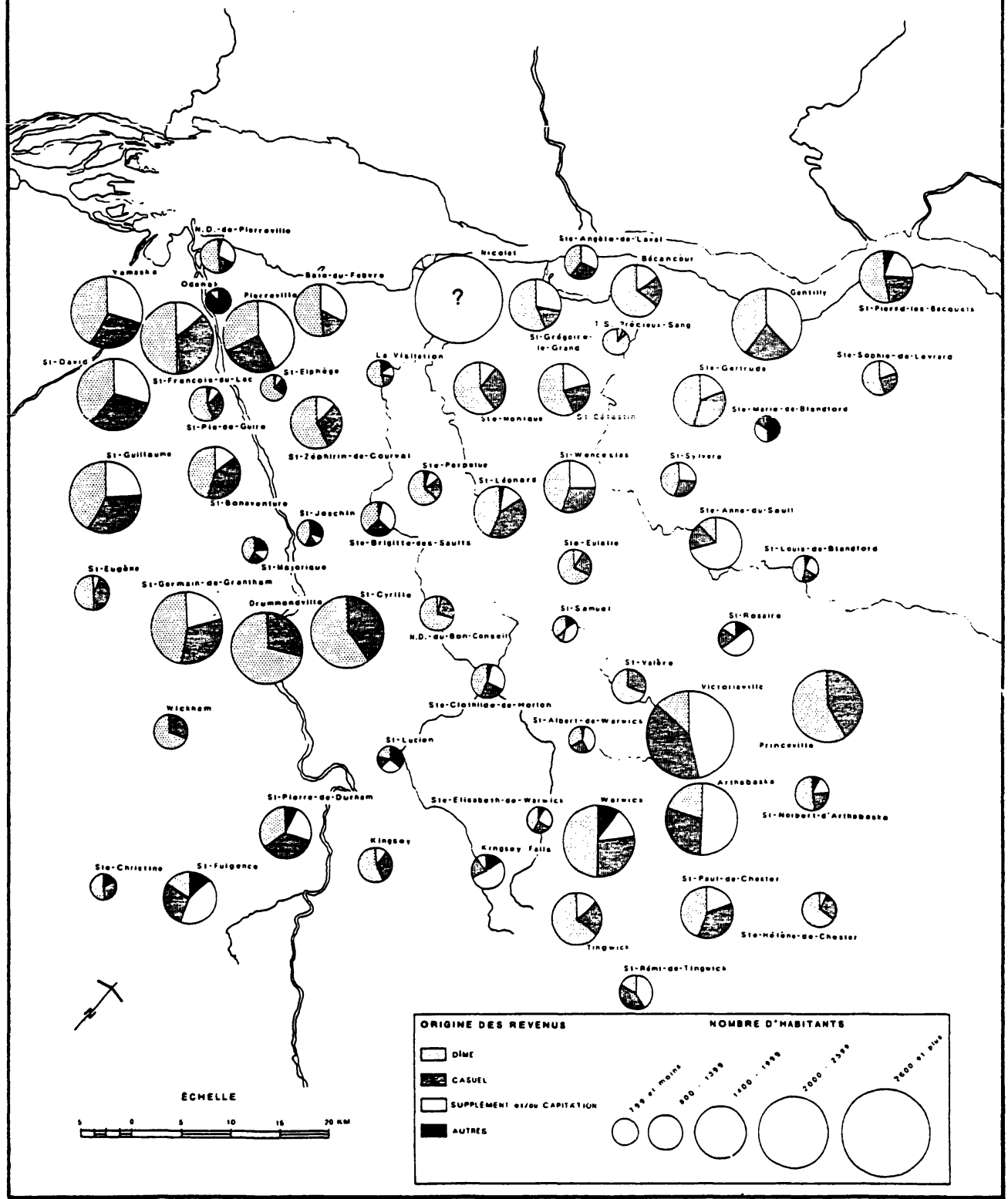


le curé de ses revenus. Pour remédier à cette situation, l'évêque prit des mesures spéciales en 1888. Il maintint la dîme des grains et imposa une taxe sur le foin, désormais versée en argent et équivalent à la valeur de «deux bottes par cent, sur le foin vendu». Le prix de vente correspondrait au montant à payer, et celui-ci serait versé aussitôt que l'acheteur aurait payé. Il serait superflu de multiplier les exemples, la répétition des interventions localisées conduisait assurément à la règlementation générale de septembre 1891. Tentons maintenant d'en mesurer les effets.

Nous avons vu qu'il y eut un réel relèvement des revenus. Il faut maintenant repérer les facteurs qui ont concouru à ce résultat. La répartition des revenus d'après leur origine devrait nous éclairer. La dîme, le casuel, le supplément et/ou la capitation et tous les autres revenus confondus ont été cartographiés. Rien de moins précis que ces «autres revenus». Ils incluent les revenus générés par l'exploitation de la terre de la fabrique. les sommes versées par l'Oeuvre de la Propagation de la Foi aux paroisses les moins bien pourvues: Sainte-Marie-de-Blandford, Saint-Rosaire, SaintJoachim-de-Courval et la mission des Abénakis recevaient 100 dollars chacune. A Odanak, le gouvernement versait 235 dollars. Dans la paroisse de Saint-Rosaire les quêtes dont celle de l'Enfant-Jésus procuraient 60 dollars. L'évêque donnait 125 dollars au curé de Saint-Lucien, auxquels s'ajoutait la recette provenant de la visite de la paroisse; ensemble ils comptaient pour $36 \%$ du revenu total de la cure. Ces paroisses étaient de création récente. Mais toutes les nouvelles paroisses n'étaient pas dans ce cas et se passaient bien de ces apports.

La dîme, c'est-à-dire le prélèvement sur les grains, était-elle devenue sans importance? Les plaintes des curés, les interventions des marguillers qui, comme à Saint-Thomas-de-Pierreville, au mois de juin 1886. attirèrent l'attention de l'évêque «sur le fait que près d'un tiers des paroissiens n'étaient pas cultivateurs, ne contribuant en aucune manière au soutien du curé» pourraient le laisser croire. Les règles édictées par l'évêque sont susceptibles de donner la même impression. Le contraire est plus exact. En 1904, 33 paroisses faisaient partie de cette classe pour qui la dîme comptait pour 40 à $60 \%$ de tous les revenus. Vingt-deux d'entre elles étaient situées dans les deux comtés en bordure du fleuve. Treize autres avaient un pourcentage encore plus élevé. Vingt ans plus tôt, une majorité importante de paroisses retiraient de la dîme plus de $60 \%$ de leurs revenus. La situation était encore plus nette vers 1875,21 curés ayant eu un revenu à $80 \%$ tiré de la dîme. Il est ainsi permis de dire qu'un glissement progressif s'était produit, déjà perceptible en 1885, mais évident en 1904. On serait donc en droit de conclure que les ordonnances ont produit leurs effets - essentiellement, que le supplément de foin ainsi que la capitation ont fait la diffé- 
rence. Voyons de plus près. À vrai dire, c'est sans surprise que l'on voit 50 cures pour qui le supplément et/ou la capitation représentaient moins de $40 \%$. Plus significatif sans doute est le fait que, pour 20 d'entre elles, ils rapportaient moins de $20 \%$ et moins de $5 \%$ dans cinq paroisses du comté de Drummond dont la paroisse de Saint-Frédéric qui avait peine à se remettre, comme le dit le procès-verbal de la visite pastorale de 1898, éprouvée qu'elle était depuis quelques années par la perte de ses industries et par les incendies.

Il ne parait pas que le supplément et la capitation puissent seuls expliquer la hausse des revenus, comme le laisse soupçonner l'analyse des pourcentages. En réalité, il faut chercher ailleurs, du côté du casuel qui, dans 46 cas, procurait entre 15 et $35 \%$ du revenu et quelquefois davantage, notamment dans quatre paroisses du comté d'Arthabaska. Il augmenta principalement après 1885 . Là dessus nous ne connaissons pas d'énoncé de politique. Il faut examiner la situation cas par cas pour finalement dégager la politique de tarification. À cet effet, il ressort de la lecture des procèsverbaux des visites pastorales que le tarif du casuel était particulier aux paroisses; il était établi en fonction des capacités de payer. Au mois de juin 1900, l'évêque coadjuteur fit la visite dans la paroisse de Sainte-Eulalie. Il ordonna que le tarif qu'il venait d'approuver soit désormais le seul en vigueur: il l'estimait proportionné aux moyens des paroissiens. Il mettait cette paroisse «sur le même pied que les paroisses bien organisées, laissant chacun libre de choisir la classe qu'il voudra». L'année suivante, il l'appliqua à la paroisse de Sainte-Anne-du-Sault. Il est certain que l'évêque était à la recherche d'une certaine uniformité. Il est tout aussi assuré que les revenus curiaux furent gonflés par l'apport occasionné par la hausse du tarif du casuel, révisé dans plusieurs paroisses au cours des années 1898-1901. En cherchant à combler le manque à gagner des fabriques, il toucha du même coup les revenus des cures.

\section{Conclusion}

Pour être plus précise, cette étude des revenus curiaux aurait dû s'appuyer sur un dossier mieux étoffé des activités économiques pratiquées sur le territoire du diocèse. À vrai dire, un tel dossier n'existe pas. Cela ne signifie pas, pourtant, que nous n'ayons rien. Les monographies qui portent sur l'industrie liée au secteur du bois, comme la compagnie Tourville ${ }^{9}$, ou au secteur de la métallurgie, comme les Forges McDou-

\footnotetext{
${ }^{9}$ Alain Gamelin, La compagnie des moulins à vapeur de Pierreville (1866-1906). Mémoire de maîtrise en Études québécoises, U.Q.T.R., 1980.
} 
gall $^{10}$, de même que les enquêtes sur l'agriculture dans le comté de Nicolet ${ }^{11}$ permettent de comprendre la démarche des autorités religieuses. Ici, les cultivateurs qui exploitaient des sols inégalement riches et qui tentaieht de s'insérer dans le courant commercial dominant; là, les ouvriers, les artisans, les professionnels qui vivaient dans des villages ou encore dans des villes naissantes ${ }^{12}$. Le tout se plaçait sous le signe de changements structuraux de l'économie.

L'analyse des statistiques démographiques avait justement pour but d'en rendre compte. Elle a fait principalement ressortir la mobilité vià les migrations régionales, la consolidation des villages, la concentration des ouvriers. Ce mouvement qui, certes, trouve son origine avant 1885 , produisit des effets nettement ressentis au cours des décennies qui nous occupent. Il ne manqua pas d'engendrer des difficultés devant lesquelles l'administration diocésaine a réagi. Or, les différences étaient telles entre les paroisses que la meilleure politique fut de ne pas en avoir, sauf à appliquer, ici et là, les mesures appropriées, d'où la succession des règlements locaux imposés dans les paroisses du diocèse de Nicolet par Louis F. Laflèche, puis par l'évêque Gravel. Aussi, lorsque le règlement général fut imposé en 1891, il paraissait entériner une situation de fait. Les hésitations et les précautions de l'évêque de Nicolet laissent penser, toutefois, que l'application n'allait pas de soi.

Les revenus curiaux ont progressé entre 1885 et 1904 . Là où le supplément et la capitation furent appliqués à la suite d'une ordonnance locale, les effets se firent sentir. De ce point de vue, l'année 1891 n'a pas tout changé, loin s'en faut. Par contre, la capitation devenait universelle. Voilà le fait important! Il faudrait voir de plus près les résistances à l'application du supplément, et la contribution effective des journaliers qui étaient principalement visés par les nouvelles mesures. Si nombre d'entre eux échappèrent, un temps, de par leur condition difficile à l'obligation de payer «la capitation», ils fournirent le casuel qui constitua une source importante des revenus des fabriques et des curés.

\footnotetext{
${ }^{10}$ Maurice Milot termine un mémoire de maîtrise en Études québécoises sur les Forges de Drummondville.

11 Normand Séguin, «L'agriculture de la Mauricie et du Québec, 1850-1950». Revue d'histoire de l'Amérique française, vol. 35, $\mathrm{n}^{\circ} 4$ (mars 1982), pp. 537-562.

${ }^{12}$ René Hardy, Pierre Lanthier et Normand Séguin, «Les industries rurales et l'extension du réseau villageois dans la Mauricie pré-industrielle, l'exemple du comté de Champlain durant la seconde moitié du $19^{\mathrm{e}}$ siècle». Article à paraître dans les Actes du colloque. Les sociétés villageoises et rapports villes-campagnes au Québec et dans la France de l'Ouest. $X V I I^{e}-X X^{e}$ siècle. Québec, septembre 1983.
} 